\title{
Optische Stufen-Temperaturmessung für die Prozessindustrie
}

\author{
Brendel, Bernhard, Grieb, Herbert; Hilsendegen, Markus, Schorb, Herbert; von Dosky, Stefan; \\ Siemens AG Karlsruhe
}

\section{Zusammenfassung}

Die Zukunft der chemischen Industrie an hochentwickelten, kostenintensiven Standorten ist eng geknüpft an innovative Prozesse und deren Anlagentechnik. Vorgaben hinsichtlich Emissionen und die Energiepreise führen zu einer Spezialisierung der „alten“ Standorte. Neue Prozesse sind oft durch viele Edukte, die einem Reaktor zugeführt werden, spezielle Temperaturstufungen und hohe Gesamtdrücke gekennzeichnet. Die Umsetzungszeit zwischen Labor- und Produktivanlage ist kurz und mitunter entsprechend wenig spezifisches Know-how und Erfahrung im sicheren Betrieb des Prozesses verfügbar. Hier ist es sinnvoll, ortsaufgelöst in den Prozess hinein zu messen. Temperaturstufungen und auch die Entstehung von schädlichen Hot-Spots versucht man typischerweise mit Stufenthermometern zu erfassen [1]. Dies sind mehrere Meter lange Stahlrohre mit eingebauten Thermoelementen. In einer solchen Lanze mit $4 \mathrm{~mm}$ Durchmesser lassen sich derzeit ca. acht Thermoelemente montieren. Da dies für einen räumlich ausgedehnten, großen Reaktor zu wenige Messstellen sind, werden viele Lanzen eingesetzt. Die Verkabelung ist aufwändig und beim Reinigen des Reaktors verschleißen die Lanzen durch Ein- und Ausbau schnell. Außerdem ist diese Art der Temperaturmessung mangels Vielseitigkeit nicht geeignet, neue Anwendungen zu erschließen.

Deshalb wurde untersucht, ob faseroptische Messverfahren „das Zeug“ haben, klassische Temperaturmessverfahren bei bestimmten Anwendungen vorteilhaft zu substituieren. Dabei sollten die Eigenschaften der Messwerterfassung (Auflösung, Genauigkeit, Reproduzierbarkeit, Langzeitstabilität etc.) vergleichbar denen klassischer Stufenthermometer sein und dies zu einem attraktiven Preis. Die Anzahl möglicher in Reihe geschalteter Messstellen ist in jedem Fall deutlich höher und damit auch die Attraktivität der faseroptischen Messverfahren.

Sämtliche am Markt verfügbaren optischen Temperaturmessverfahren wurden analysiert und teilweise im Labor erprobt. Die Faser-Bragg-Gittermesstechnik (FBG) erscheint derzeit am weitesten entwickelt für die Zielapplikationen.

Im Beitrag wird zunächst ein Vergleich faseroptischer Messverfahren hinsichtlich deren Möglichkeiten zur räumlich aufgelösten Temperaturmessung in verschiedenen Applikationen vorgenommen. Für die gewählte FBG-Messtechnik [2, 3] wird die gängige Faser- und Auslesegerätetechnik [4] an den Forderungen der Temperaturmesstechnik gespiegelt. Eine Variante zur langzeitstabilen Referenzierung der Temperaturskala in Bezug auf das gemessene optische Signal wird erläutert, Querempfindlichkeiten zu anderen physikalischen Phänomenen und deren mögliche Ursachen werden aufgezeigt und schließlich ein vergleichsweise robustes Temperaturmesssystem zum Einsatz in explosionsgeschützten Druckbehältern beschrieben.

\section{Faseroptische Messverfahren: Stand der Technik und Auswahl}

Seit vielen Jahren ist diese Klasse von Messverfahren etabliert bei der Erfassung verschiedenster physikalischer Effekte entlang einer optischen Faser. Größen wie Temperatur, Dehnung oder Brechungsindex des angekoppelten Mediums (Festkörper, Flüssigkeit, Gas) sind erfassbar. Im einfachsten Fall wird Licht in eine Faser eingekoppelt und das reflektierte Licht bezüglich seiner Änderung der Frequenz und ggf. Amplitude und Phase analysiert. Die physikalischen Effekte bewirken bei den etablierten Messverfahren die folgenden Änderungen der Messsignale (Bild 1) 
- Faser-Bragg-Gitter (FBG): Auslesegeräte werden typischerweise mit polychromatischer Lichtquelle betrieben. Jedes Gitter ist ein schmalbandiger Reflektor in einem kleinen Volumenelement der Faser, dessen Mittenfrequenz sich durch einwirkende physikalische Größen ändert. Die Signalamplitude kann ggf. zur Sensordiagnose eingesetzt werden. Die Anzahl möglicher Sensoren pro Faser hängt u. a. von der Bandbreite des Auslesegerätes ab und ist meist $<100$.

- Optical Backscatter Reflectometry (OBR): Das Ausleseverfahren verbindet FBG- und OFDRTechnik. Die Amplitude des rückgestreuten Rayleigh-Spektrums verändert sich lokal entlang der Faser beispielsweise durch Änderung der Temperatur. Der Messeffekt tritt an jeder Faser auf. Das Reflexionsspektrum bei bekannter Temperatur wird mit demjenigen bei veränderlicher Temperatur vergleichen. Der Effekt kann durch eingeschriebene FBGs (mehrere Tausend) derselben Wellenlänge in eine Frequenzmessung verändert werden.

- Optische Zeitbereichs-Reflektometrie (OTDR): Auslesegeräte haben einen Pulslaser als Lichtquelle. Gemessen wird die Lichtlaufzeit (Messort) sowie die Amplitude des jeweiligen Raman-Spektrums (Amplitudenverhältnis Stokes - Antistokes).

- Optische Frequenzbereichs-Reflektometrie (OFDR): Auslesegeräte haben eine Laserlichtquelle mit durchstimmbarer Amplitudenmodulation. Gemessen wird das zurückgeworfene Amplitudenspektrum über der Modulationsfrequenz, aus dem durch Fourier-Rücktransformation jeweils der ortsabhängige Verlauf des Stokes- und des Antistokes-Signals berechnet wird und durch Quotientenbildung schließlich der Temperaturverlauf resultiert.

OBR ist als Temperaturmessgerät für Fasern $<500$ m Länge technisch vergleichsweise aufwändig. OTDR und OFDR sind neben dem Raman- auch für das Brillouin-Spektrum erhältlich. Während das Brillouin-Verfahren gleichermaßen für Druck und Temperatur empfindlich ist, zeigt das Raman-Prinzip nur eine geringe Druckabhängigkeit. Die Messorte (Sensoren) entsprechen bei diesen Verfahren jeweils $<1 \mathrm{~m}$ langen Fasersegmenten. Beide Messverfahren ermöglichen jedoch mehrere zehntausend Messstellen pro Faser, die bis zu $50 \mathrm{~km}$ lang sein kann [5]. Anwendungen sind typischerweise die Überwachung räumlich ausgedehnter Objekte (Pipelines, Tunnel etc.)

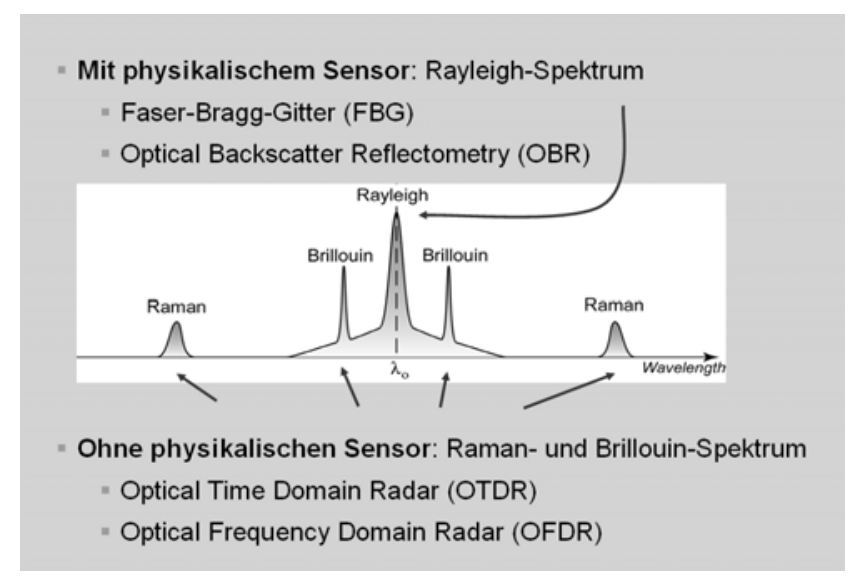

Bild 1 Temperaturmessverfahren mit Sensoren entlang einer Glasfaser und der beeinflussten spektralen Bestandteile des rückgestreuten Lichtes 
Die Möglichkeit der robusten Temperaturmessung mit vielen Sensoren wird am ehesten vom FBGMessverfahren erfüllt. Dieses schon seit langem bekannte Verfahren ermöglicht die Fertigung vergleichsweise ausgereifter und mit preisgünstiger Telekom-Hardware (1500-nm-Bänder) ausgerüsteter Auslesegeräte. Bei den Sensoren wird grob zwischen Standard-FBGs und Zugturm-FBGs unterschieden. Die reflektierte Lichtmenge von Zugturm-FBGs ist durch die kurze Einschreibzeit des Bragg-Gitters deutlich geringer (ca. Faktor 10) als bei Standard-FBGs. Außerdem sind Zugturm-FBGs zwingend mit einer Beschichtung (Cladding) zu versehen, die aus Kunststoff besteht und bis max. ca. $250{ }^{\circ} \mathrm{C}$ im Dauerbetrieb belastbar ist. Dafür ist die Herstellung vergleichsweise preiswert.

\section{Konfiguration des Messsystems für Branchenanforderung}

Nach der Recherche war klar, dass mit einigem gerätetechnischen Aufwand sämtliche Messsysteme <0,5 K auflösen, sich mit einigen Systemen Temperaturprofile über dutzende Kilometer erstellen lassen und ein Dauertemperaturbereich von -270 bis $+600{ }^{\circ} \mathrm{C}$ abgedeckt werden kann. Mit FBGs in Saphirfasern sind Temperaturmessungen $>1000{ }^{\circ} \mathrm{C}$ möglich. Für die eigene Erprobung wurde jedoch ein System konfiguriert, das viele Applikationen beispielsweise in der chemischen Industrie ermöglicht. Typische Anforderungen sind hier: Messbereich -40 bis $+400{ }^{\circ} \mathrm{C}$; Auflösung $0,1 \mathrm{~K}$; Länge des Messortes $<100 \mathrm{~mm}$; kein Übersprechen der Messorte im Messsystem, Länge des Profils $<30 \mathrm{~m}$; Genauigkeit $1{ }^{\circ} \mathrm{C}$; Robustheit, Auslesefrequenz und Langzeitstabilität vergleichbar mit PT100; Messstellen dort, wo Hot-Spots erwartet werden; optische Leistung in Faser $<30 \mathrm{~mW}$; Preis pro Messstelle vergleichbar mit demjenigen von konventioneller Punktsensorik.

Für den Einsatz der vergleichsweise aufwändigen Messverfahren ohne physikalischen Sensor und des OBR ergab sich somit keine Notwendigkeit. Vielmehr entwickelte sich seitens des Auslesegerätes das Ziel, den Weg zu einem kompakten Hutschienenmodul aufzuzeigen, das sich nahtlos in eine Automatisierungslösung einfügt. Seitens des Sensors bewältigt eine Messlanze (Faser in einem langen Edelstahl-Kapillarrohr) die meisten Anforderungen.

Es wurde zunächst eine $5 \mathrm{~m}$ lange Lanze mit 25 Zugturmgittersensoren gebaut und in den Reaktorbehälter einer Demonstrationsanlage mit einem Durchmesser von $500 \mathrm{~mm}$ schraubenförmig eingewickelt. Die IstWellenlängen nach Einbau wurden bei einer konstanten Temperatur als Nullpunkt vermerkt. Der Behälter wurde jeweils über mehrere Tage abwechselnd mit kaltem und heißem Wasser gefüllt und das Wasser wurde jeweils vor einer Messung verrührt, sowie die Temperatur mit einem Referenzmessgerät erfasst. Mit dieser Anordnung lässt sich vergleichsweise gut der Behälterfüllstand erfassen - zur Messung der Wassertemperatur reicht diese Anordnung jedoch nicht aus. Es wurde festgestellt, dass nach mehreren Tagen und vielen Wechselzyklen jeweils im stationären Zustand die aus der Wellenlänge umgerechnete Temperaturdifferenz der 25 Sensoren untereinander $5 \mathrm{~K}$ beträgt. Die größte Abweichung eines einzelnen FBGs von der PT100-Referenz betrug 6 K. Dies wird den genannten Anforderungen natürlich nicht gerecht. Um das ungünstige Messverhalten zu verbessern, wurde die Faser in zwei alternativen Kapillarrohren untersucht. Die Außendurchmesser sind typischerweise 1/16" und die Innendurchmesser wurden mit 0,75 und $1 \mathrm{~mm}$ gewählt, so dass eine $12-\mu \mathrm{m}$-Faser makroskopisch mechanisch nicht klemmen kann. Rohrknicke wurden beim Einwickeln in den Behälter vermieden. In diesem Zusammenhang wurde auch die Rautiefe der Innenfläche des Rohres bestimmt, die jeweils $\mathrm{Rz}<20 \mu \mathrm{m}$ war. Weitere Tests mit geradem, senkrecht eingebautem Schutzrohr wurden durchgeführt. Dabei war sichergestellt, dass die Faser nicht auf dem Rohrinnendurchmesser aufliegt. Die Messunsicherheit konnte dadurch zwar verringert werden, aber trotzdem wurden Irreversibilitäten identifiziert. Die finale Begründung wird im Compound Faser/Cladding vermutet. Das verwendete Ormocer-Cladding [7] hat im Temperaturbereich -60 bis $+85{ }^{\circ} \mathrm{C}$ einen höheren Dehnübertragungskoeffizienten [6] zum Stahlröhrchen (Äquivalent zur Rauhigkeit) als Glas. Eine mögliche Kraftübertragung auf die Faser beim Anhaften an der Rohrinnenwand ist im Fall Ormocer größer. Weiterhin entsprechen sich die thermischen Ausdehnungskoeffizienten von Ormocer (angegeben mit 17E-6 1/K) und dem von Gläsern nicht (gemein hin <10E-6 1/K, die genaue Glaszusammensetzung war allerdings nicht bekannt). In jedem Fall werden über den weiten Temperaturmessbereich (getestet bis $150{ }^{\circ} \mathrm{C}$ ) Kräfte zwischen Cladding und Faser erzeugt. Durch den Haftreibverbund (UV-Härtung nach Beschichtung) ist es vorstellbar, dass dies Kräfte nichtlinearer Wirkung sein könnten. Zusammenfassend sind die bei den beschichteten Zugturmfasern im Kapillarrohr gemessenen Wellenlängen-Hysteresen über das jeweils einwirkende Temperaturkollektiv für ein verteiltes Temperaturmessgerät nicht tolerierbar. 
Parallele Untersuchungen einer Lanze ähnlichen Durchmessers mit einer unbeschichteten Glasfaser (3 m lang, zehn Standard-FGBs) zeigten diese negativen Effekte nicht. Der Nachteil dieser Anordnung ist jedoch die zu erwartende geringere mechanische Beanspruchbarkeit der Lanze.

Seitens möglicher Auslesegeräte wurden ein Spektrometer-basiertes (Quelle=Superlumineszenzdiode, Senke=Spektrometer) und ein Frequenz-scannendes System (Quelle=Wobbel-Laser, Senke=Photodiode) untersucht. Die messtechnischen Eigenschaften sind laut Datenblatt - außer der Auslesefrequenz - ähnlich. Diese spielt jedoch für die Temperaturmessung bei den ohnehin vorhandenen thermischen Trägheitszeitkonstanten keine Rolle. Es wurde ermittelt, dass das Gerät der Spektrometer-Kategorie eine mittlere Einschaltzeit bei Raumtemperatur von ca. drei Stunden hat, nach der die gemessenen Mittenwellenlängen konstant sind bei konstanter Lanzentemperatur. Bei der Drift in diesem Intervall wurden sowohl positive als auch negative Gradienten der Mittenwellenlängen gemessen. Die Änderung während dieser Aufwärmzeit betrug bis zu 15 pm, was ca. 1,5 K entspricht. Nach dieser Zeit sind dann die Mittenfrequenzen konstant, wobei in verschiedenen Versuchen über jeweils mehrere Tage gemessen wurde. Der gemessene Wellenlängen-Offset zwischen zwei Einschaltvorgängen beträgt bis zu $30 \mathrm{pm} \cong 3 \mathrm{~K}$. Vom Betrag her ähnliche Drifteffekte wurden bei der sprungförmigen Änderung der GeräteUmgebungstemperatur (Kondensation wurde ausgeschlossen) beobachtet. Die Driftursache wurde mit dem Hersteller lokalisiert, ist aber offensichtlich bauartbedingt für diese Geräteklasse und nicht kompensierbar. Das Gerät der Laser-Kategorie liefert stets nach ca. fünf Minuten konstante Mittenwellenlängen ohne relevanten Offset, weshalb es bevorzugt wurde.

Weiterhin wurde eine langzeitstabile Referenzierung des Auslesegerätes erarbeitet. Ein Referenzmodul koppelt thermisch ein kalibriertes PT100 und ein FBG in einer isolierten Einheit, die sich im Schaltschrank befindet. Dieses Modul wird zyklisch vom Auslesegerät abgefragt. Da das Referenz-FBG keinen schädlichen Umwelteinflüssen und nur moderaten Temperaturänderungen ausgesetzt ist, liefert es eine langzeitstabile Mittenfrequenz bezogen auf die Messunsicherheit des PT100 (30 mK). Die resultierende Messunsicherheit der Wellenlänge wurde nicht untersucht, sie dürfte aber im Bereich der optischen Auflösung des Auslesegerätes $(1 \mathrm{pm} \cong 0,1 \mathrm{~K})$ liegen. Durch den geringen Temperaturmessbereich der Referenz ist auch eine deutlich bessere Polynom-Approximation als für die Lanze durchführbar (Bild 2).

\section{Exemplarische Messergebnisse}

Zur Ermittlung der Temperatur aus der gemessenen Wellenlänge werden Polynome höherer Ordnung benutzt. Für kleinere Messbereiche ist ein Polynom zweiter Ordnung noch ausreichend, [6]. Soll jedoch der durch die Lanze nutzbare Messbereich von -50 bis $+700{ }^{\circ} \mathrm{C}$ ausgeschöpft werden, ergeben sich damit zu große Approximationsfehler, Bild 2. Daher wurde für die nachfolgenden Messungen die vierte Polynomordnung gewählt, die im Messbereich $>30^{\circ} \mathrm{C}$ geringe systematische Fehler erzeugt.

Die jeweiligen Stützstellen sind Referenzwerte, die durch statistische Auswertung eines Ensembles verschiedener FBG-Temperatursensoren über längere Messphasen und in verschiedensten Applikationen ermittelt wurden.

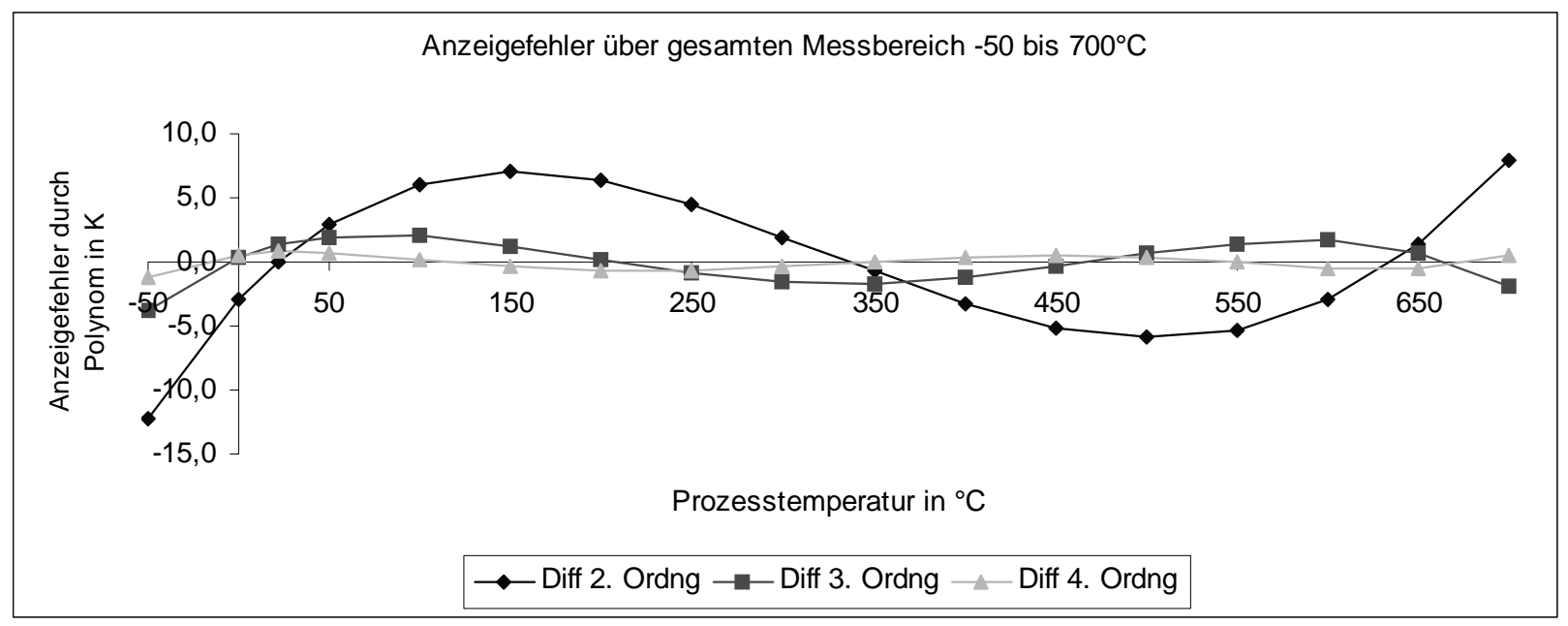




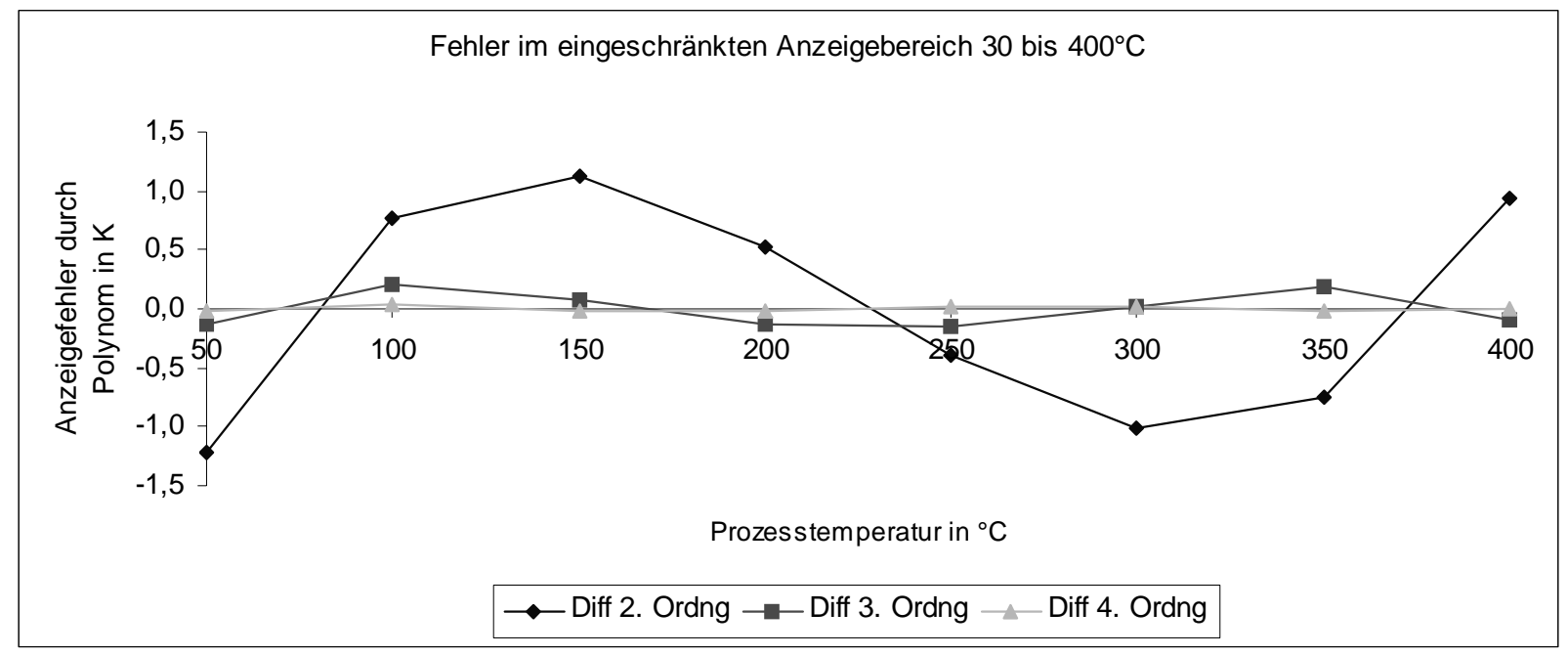

Bild 2: Berechnete Anzeigefehler durch Approximation einer Look-up-Tabelle, erweiterter und eingeschränkter Anzeigebereich

Exemplarische Messergebnisse des Systems mit laserbasiertem Auslesegerät mit unbeschichteter Glasfaserlanze zeigen Bilder 3 und 4.
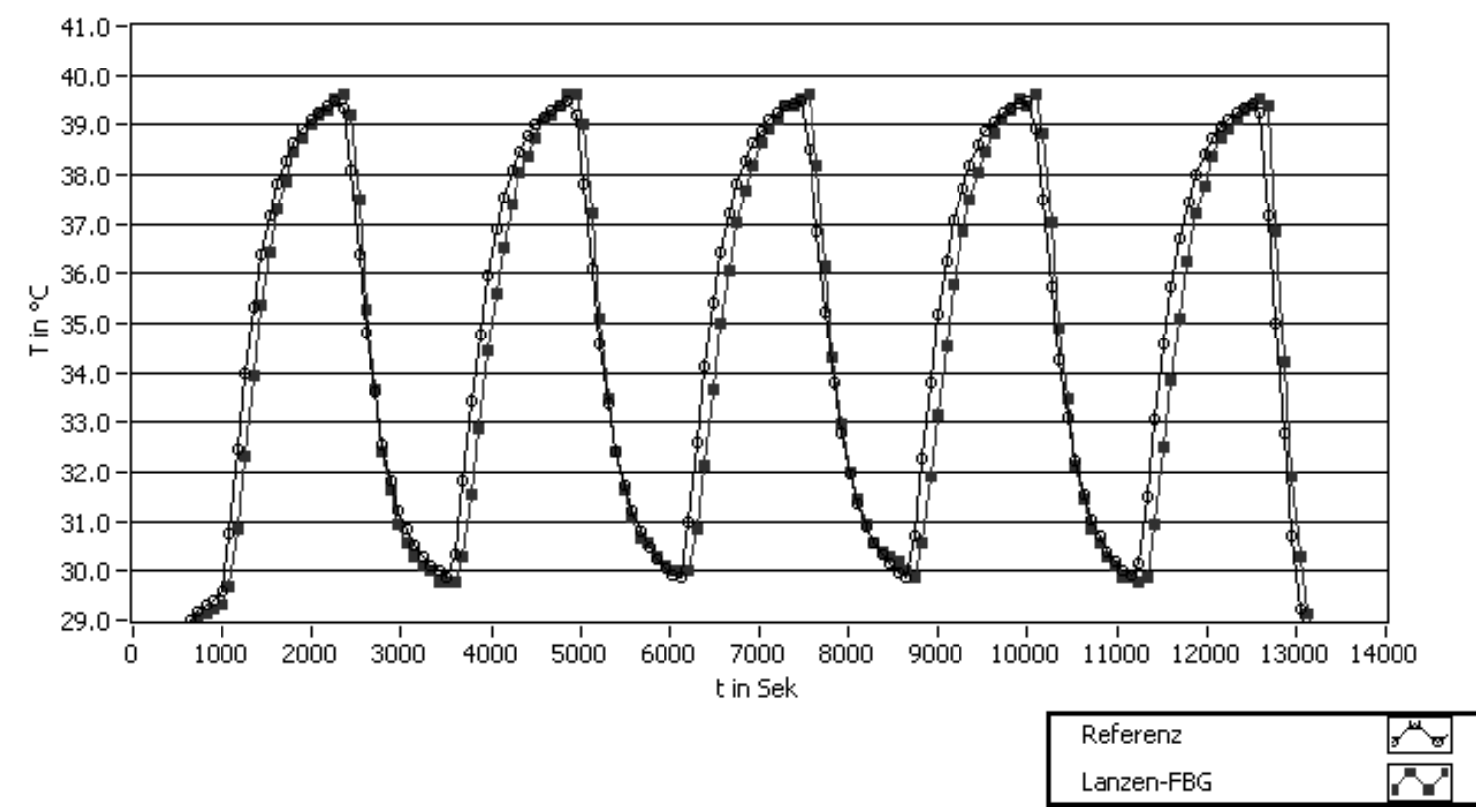

Bild 3: Reproduzierbarkeit eines FBG-Temperatursensors in der Messlanze 


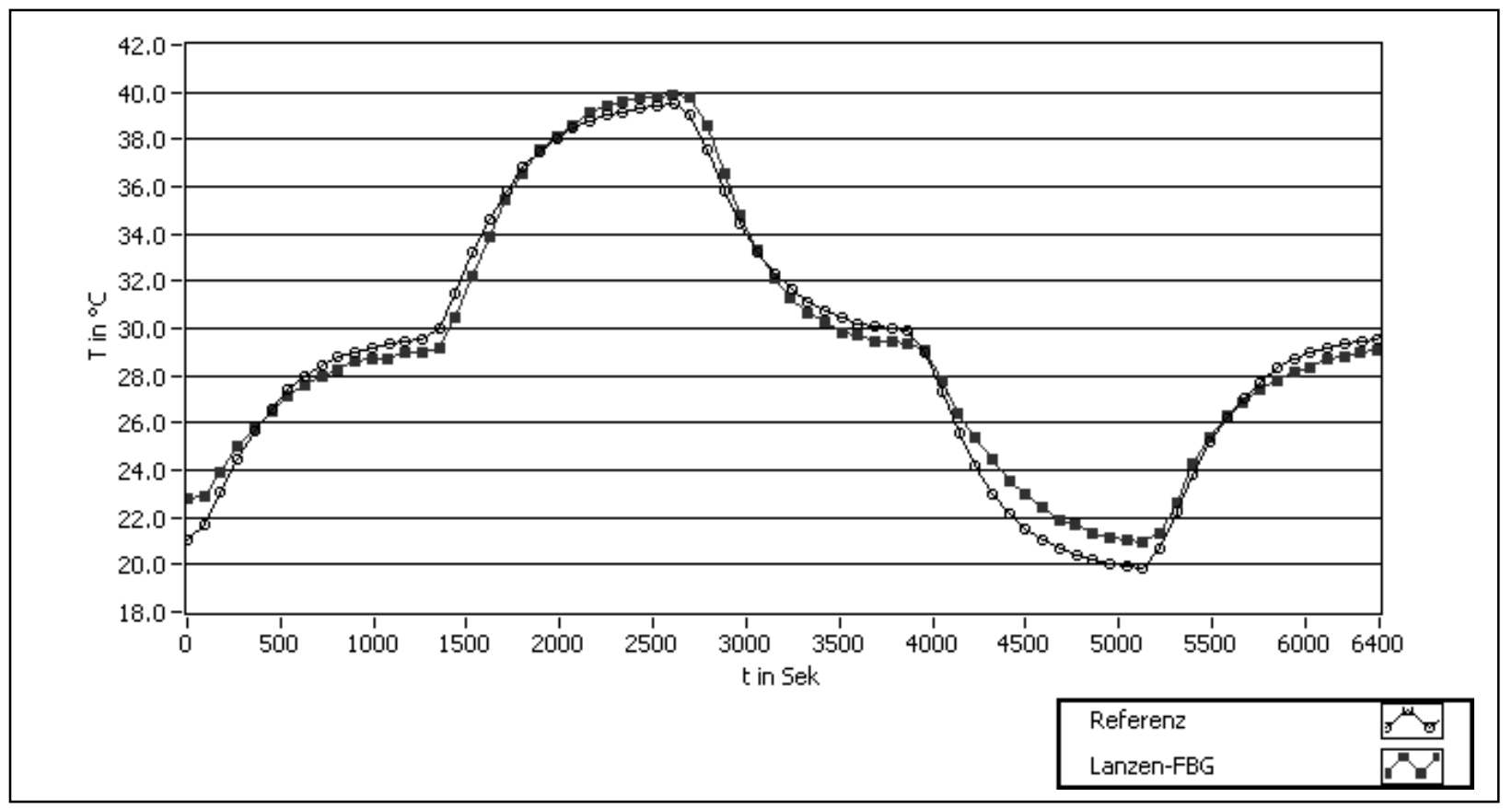

Bild 4 Messfehler beim Durchfahren einer Temperatur-Hysterese: 1,1K sind im Polynom-fit begründet, der im Bereich der Raumtemperatur vergleichsweise ungenaue Ergebnisse liefert (Bild 2).

Weiterhin wurden Hot-spot-Tests mit einer Heißluftquelle durchgeführt und das stark aufgeheizte Lanzensegment jeweils wieder auf die Referenztemperatur (Badthermostat) gekühlt. Die exemplarische Messunsicherheit eines Lanzen-FBGs konnte bei dieser Prüfung mit $+/-0,6{ }^{\circ} \mathrm{C}$ ermittelt werden. Die Einstellzeit T90 erwies sich als vergleichsweise klein und beträgt $<4$ Sekunden. Ähnliche Messergebnisse konnten mittlerweile auch bei horizontalem Einbau der geraden, unbeschichteten Lanze ermittelt werden.

Nach Lieferung und Einbau der Lanze in das zu messende Objekt muss nur noch eine Offset-Korrektur mit einer beliebigen Referenztemperatur gemacht werden. Eine Werkskalibrierung ist bei diesem System nicht erforderlich.

\section{Literatur}

[1] Siemens AG: Stufenthermometer

[2] AOS GmbH, www.aos-fiber.com

[3] IPHT Jena, www.ipht-jena.de

[4] Fibersensing S.A.: Auslesegerät Braggmeter

[5] Omnisens, www.omnisens.com

[6] Schlüter, V.G., Entwicklung eines experimentell gestützten Bewertungsverfahrens zur Optimierung und Charakterisierung der Dehnungsübertragung oberflächenapplizierter Faser-Bragg-Gitter-Sensoren, Dissertation TU-Berlin, 2010

[7] www.ormocer.de 